\title{
Electrochemical Reduction Potential Shifts of Graphene Oxide Employed in Thrombin Detection
}

\author{
Hanall Jeong, Shinjae Hwang, and Kyuwon Kim* \\ Department of Chemistry, Incheon National University, Incheon 406-772, Korea. *E-mail: kyuwon_kim@incheon.ac.kr \\ Received January 18, 2014, Accepted February 18, 2014
}

Key Words : Graphene oxide, Electrochemical reduction, Thrombin detection, Aptamer, Electrochemical inpedance

The development of electrochemical biosensor is currently under the intense investigation owing to their great promise for rapid, convenience and low-cost detection of analytes. Especially, graphene oxide (GO), an oxygen-rich carbonaceous layered material, has attracted strong interest as an substrate material because of its own unique characteristics. ${ }^{1-3}$ Based on recent studies GO consists of intact graphitic regions interspersed with $\mathrm{sp}^{3}$-hybridized carbons containing hydroxyl and epoxy functional groups on the top and bottom surfaces of each sheet and $\mathrm{sp}^{2}$-hybridized carbons containing carboxylate and carbonyl groups principally at the sheet edges. ${ }^{4-7}$ The intrinsic oxygen-containing functional groups have been used as initial sites for deposition of biomolecules such as DNA and proteins on the GO sheets and provide the possibility to be an ideal platform for detecting of target materials. ${ }^{89}$ In addition, GO is electrochemically reducible, ${ }^{10-12}$ which enables it as an indicator or label for electrochemical sensor applications. Several studies have employed the GO-indicator to determine DNA, $\mathrm{Hg}^{2+}$, and thrombin. ${ }^{13-15}$

Here, we for the first time report that the peak potential shifts for the electrochemical reduction of GO could be used in the determination of target molecules. GO-adsorbed electrode surfaces were derivatized with thrombin-binding aptamer (TBA). TBA is one of a family of DNA oligomer that binds specifically to thrombin with high affinity. ${ }^{16,17}$ We found that the reduction potential of GO in the TBAmodified surfaces shifts more positively upon the adsorption of thrombin. The magnitude of the shifts was increased with increasing thrombin concentration, which could be employed as an signal for detection of thrombin as a target protein.

Scheme 1 shows that a procedure to fabricate the TBAmodified surfaces and a scheme for electrochemical thrombin detection. A bare gold electrodes was modified with

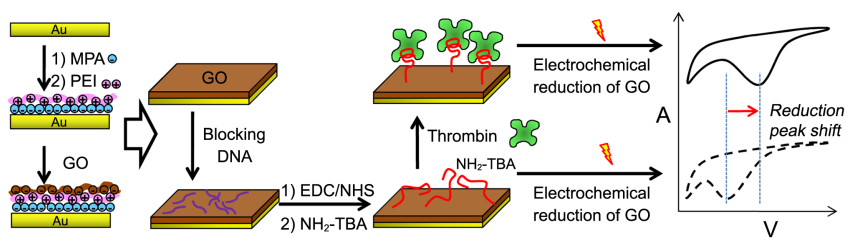

Scheme 1. A schematic illustration of process to fabricate TBAmodified surfaces and electrochemical thrombin detection.
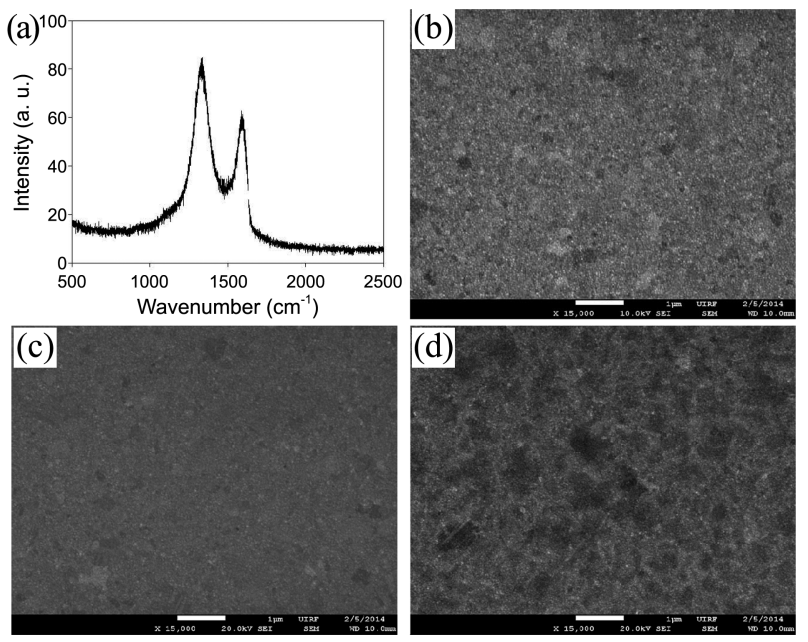

Figure 1. (a) Raman spectra of graphene oxide (D band: 1328 and G band: 1586, respectively). SEM images of (b) bare gold, (c) PEImodified gold, and (d) GO-treated PEI-modified gold surface.

mercaptopropionic acid (MPA) through a self-assembly process to make negative charge-ended MPA/Au surface. The resulting surface was used to immobilize a positively charged polyethyleneimmine (PEI). A negatively charged GO sheet in aqueous medium was adsorbed onto the positively charged PEI/MPA/Au through electrostatic interaction. After then, we used a mixed solution of $N$-hydroxysuccinimide (EDC) and $N$-(3-Dimethylamino-propyl)- $N$ 'ethylcarbodiimide hydrochloride) (NHS) to activate carboxylic acid on the surface of the attached $\mathrm{GO}$, producing the activated ester. $\mathrm{NH}_{2}$-terminated TBA $\left(\mathrm{NH}_{2}-\mathrm{C}_{6}-5\right.$ 'TTT TTT TTT TTT TTT AGT CCG TGG TTG GTG TGG TTG GGG TGA CT-3') was immobilized onto GO/PEI/MPA/Au through the covalent coupling reaction. ${ }^{18,19}$ Thrombin may be captured by TBA through forming G-quadruplex structure, which changes a chemical circumstance of GO, resulting in the different electrochemical behavior of GO (Scheme 1).

GO was characterized with atomic force microscopy (AFM), which confirms single-layer GO sheets with $\sim 1 \mathrm{~nm}$ thickness. ${ }^{12}$ Raman spectroscopy for GO exhibited a typical D/G peak ratio, as shown in Figure 1(a). ${ }^{4}$ Scanning electron microscope (SEM) images confirms the immobilization of GO. The distinctive pattern of GO-immobilized surface 

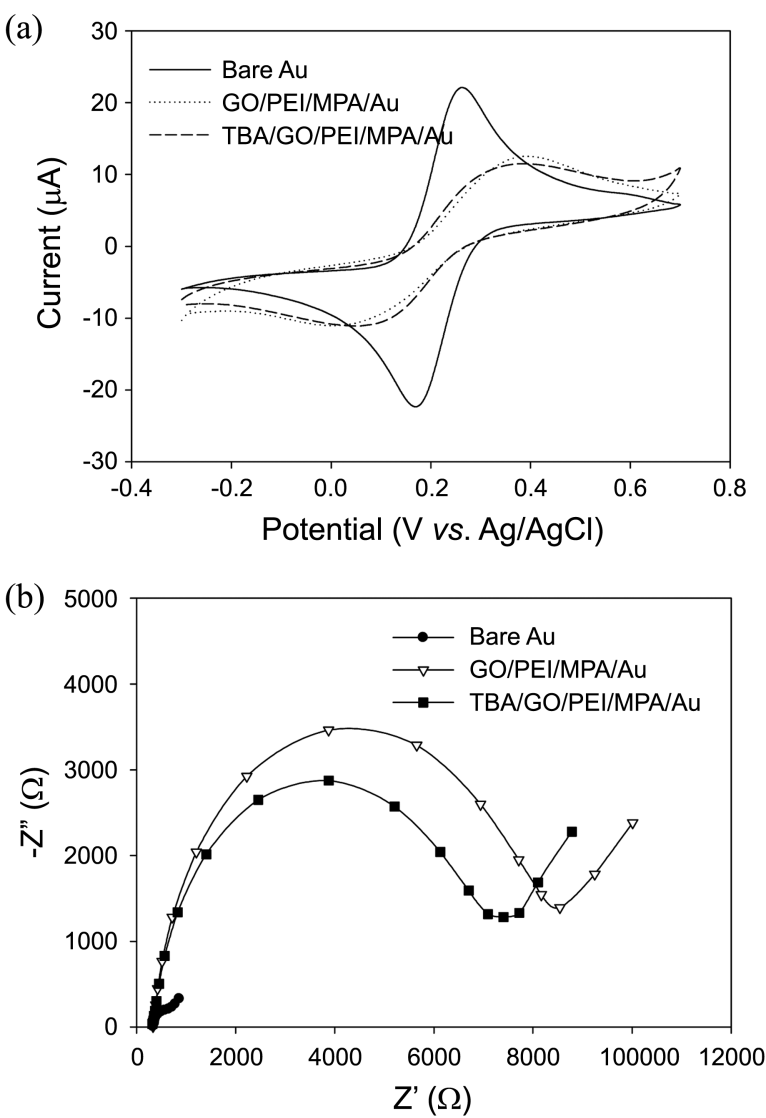

Figure 2. (a) CVs and (b) Nyquist plots obtained for bare, GOmodified and TBA-treated GO-modified gold electrode in $1 \mathrm{mM}$ $\mathrm{K}_{3}\left[\mathrm{Fe}(\mathrm{CN})_{6}\right] / \mathrm{K}_{4}\left[\mathrm{Fe}(\mathrm{CN})_{6}\right]$ solution at a scan rate of $50 \mathrm{mV} / \mathrm{s}$.

(Figure 1(d)) compared to the bare gold (Figure 1(b)) and the PEI-modified surface (Figure 1(c)) signified stably adsorbed GO. The electrochemical interfacial features resulting from the chemical reactions on GO/PEI/MPA/Au were characterized by cyclic voltammetry (CV) and electrochemical impedance spectroscopy (EIS). CV and EIS were carried out with a redox couple of $\mathrm{Fe}(\mathrm{CN})_{6}{ }^{3-}$ and $\mathrm{Fe}(\mathrm{CN})_{6}{ }^{4-}$. As shown Figure 2(a), the surface GO/PEI/MPA/Au showed a larger peak separation and smaller peak current in the $\mathrm{CV}$ for the redox couple than the bare gold electrode due to the insulating properties of GO and the repulsive interactions between the negatively charged GO sheets and the redox couple, as well as steric effect. Figure 2(b) display that the charge transfer resistance $\left(\mathrm{R}_{\mathrm{ct}}\right)$ investigated with EIS was dramatically increased at the GO-modified surface from that on the bare gold electrode, which is consistent with the $\mathrm{CV}$ results. TBA was immobilized on GO/PEI/MPA/Au by covalent attachment procedure as mentioned above. It should be noted that DNA including TBA is generally adsorbed also on the basal plane of GO through the $\pi-\pi$ stacking interactions between the base of DNA and aromatic ring of the plane. ${ }^{12,20}$ In addition, the adsorbed TBA can be easily removed from plane upon by capturing of thrombin in solution. To prevent the unwanted adsorption, we pretreated GO/PEI/MPA/Au with a solution of DNA oligomer (5'-TTT TTG GGT TTT T-3'), named as a blocking-DNA prior to the

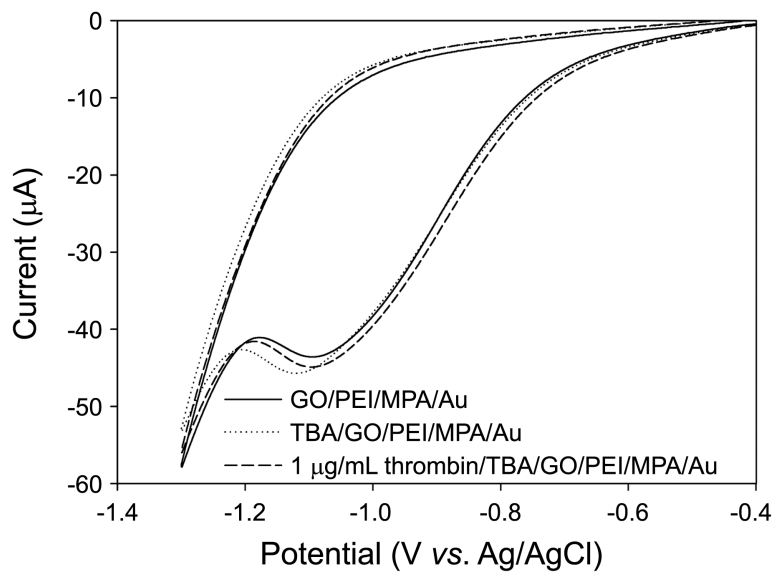

Figure 3. CVs of the electrochemical reduction of $\mathrm{GO}$ on the various GO-modified gold electrode in $20 \mathrm{mM} \mathrm{NaCl}$ solution at a scan rate of $100 \mathrm{mV} / \mathrm{s}$.

EDC/NHS activation of the GO. Because the DNA sequence is irrelevant to thrombin, the basal plane of GO could be efficiently blocked.

In CV and EIS tests, the resulting surface exhibited a slightly reduced blocking behavior compared to the surface before the immobilization (Figure 2(a) and 2(b)). This was an opposite result to the expected one, which might be attributed to partial loss of GO layer during the immersion procedure of the GO-modified surface including EDC/NHS activation and TBA immobilization.

$\mathrm{CV}$ was used to monitor the change of peak potential of the electrochemical reduction of GO before and after immobilization of TBA. Potential scanning was performed range from -0.4 to $-1.3 \mathrm{~V}$ (vs. $\mathrm{Ag} / \mathrm{AgCl}$ ), in which an electrolyte solution with a lower concentration of salt (20 $\mathrm{mM} \mathrm{NaCl}$ ) than usually employed one was used. The electrochemical redox potential shift is generally more sensitive at lower salt concentration because the shift must be affected by the charge environments of electroactive species. After the TBA immobilization, the reduction peak potential was changed by $22.3( \pm 2.2) \mathrm{mV}$ toward negative direction from the one for GO/PEI/MPA/Au as shown in Figure 3, which implies the reduction becoming more difficult. The immobilized TBA has high flexibility due to the long length of poly-Thymine as a spacer part of the chain, and the amine groups in the base of the immobilized TBA can attractively interact with the oxygen moieties of GO such as hydroxyl, carboxyl, and carbonyl groups through hydrogen bonding or electrostatic interaction. These interactions might be able to stabilize the oxygen groups in the structure of GO, resulting in the negative shifts in the reduction potential.

We observed the reduction peak potential after the exposure of TBA/GO/PEI/MPA/Au to Tris-buffer solution with various concentration of thrombin. Before the observation, thrombin-treated surfaces were subjected to the measurement of $R_{c t}$. Figure 4(a) shows $R_{c t}$ increases with increasing the concentration of thrombin (from $1 \mathrm{ng} / \mathrm{mL}$ to $1 \mu \mathrm{g} / \mathrm{mL}$ ), consistent with the previous reports. ${ }^{21,22}$ In specificity test, other proteins such as avidin, mouse IgG didn't show any 

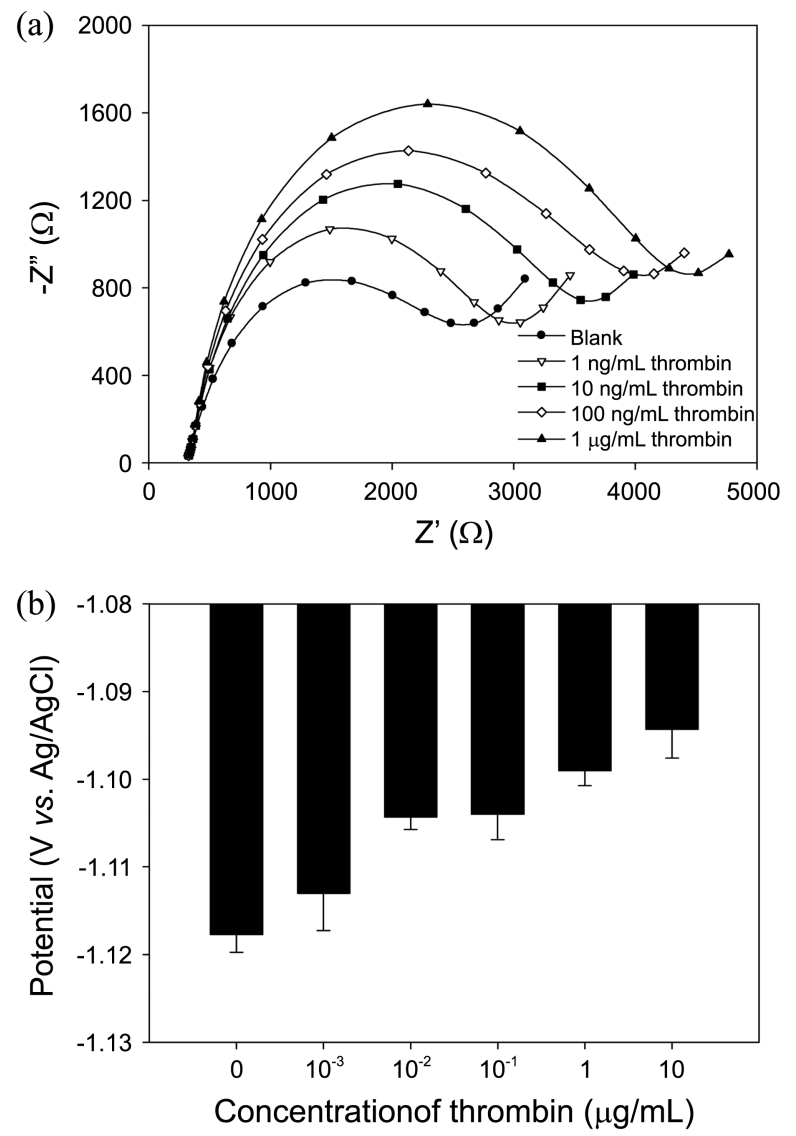

Figure 4. (a) Nyquist plots for various thrombin concentrations obtained with EIS conducted on TBA-modified GO surface on gold electrode. (b) The dependence of the reduction peak potential on the thrombin concentration.

significant changes in $\mathrm{R}_{\mathrm{ct}}$ value. These results confirmed that the TBA-modified surface can be served as a platform to detect thrombin as a target. The platform was used in the measurement of the reduction peak potential of GO. After the exposure of $1 \mu \mathrm{g} / \mathrm{mL}$ thrombin solution to the surface the reduction peak shifted to the positive direction, resulting in a similar value to the one obtained from GO/PEI/MPA/Au, as shown in Figure 3. Figure 4(b) shows thrombin concentration-dependent shifts of the peak potential from the result observed from blank solution without thrombin. The gradual positive shifts of the peak potential indicates that the formation of TBA-thrombin complex made the reduction easier, which might be attributed to a disruption of the attractive interactions between free TBA chain and the oxygen group of GO. The dependency of peak shifts has wide dynamic range of $1 \mathrm{ng} / \mathrm{mL}$ to $10 \mu \mathrm{g} / \mathrm{mL}$, suggesting that the shifts could be employed for the determination of target thrombin. The limit of detection (3SD) estimated from the data of Figure 4(b) was as low as $10.8 \mathrm{ng} / \mathrm{mL}$. In addition, we found that such trend has never been observed on TBA/ GO/PEI/MPA/Au without treatment of the blocking-DNA.

In conclusion, we have reported a feasibility study on thrombin detection by the measurement of peak potentials shifts for the electrochemical reduction of GO. Our novel strategy has demonstrated that the shifts are fairly dependent upon the concentration of target thrombin. We are extending this result to determine quantitatively various target material such as proteins, DNA, metal ions.

\section{Experimental}

TBA $\left(\mathrm{NH}_{2}-\mathrm{C}_{6}-5\right.$ 'TTT TTT TTT TTT TTT AGT CCG TGG TTG GTG TGG TTG GGG TGA CT-3') and blocking DNA (5'-TTT TTG GGT TTT T-3') were purchased from Genotech (Korea). All chemicals including graphite powder $(<150 \mu \mathrm{m})$ were purchased from Sigma Aldrich. Tris-HCl buffer consist of $25 \mathrm{mM}$ Tris- $\mathrm{HCl}$ and $300 \mathrm{mM}$ and $1 \mathrm{M}$ $\mathrm{NaCl}$ with $\mathrm{pH}$ 7.4. Phosphate buffer saline (PBS) consists of $100 \mathrm{mM}$ phosphate and $100 \mathrm{mM} \mathrm{NaCl}$. The binding buffer consists of $50 \mathrm{mM}$ Tris- $\mathrm{HCl}, 140 \mathrm{mM} \mathrm{NaCl}, 1 \mathrm{mM} \mathrm{MgCl}$, and $0.1 \%$ bovine serum albumin (BSA). All electrochemical measurements, including $\mathrm{CV}$ and EIS, were carried out using CHI-A617 and Ivium Compactstat as a potentiostat interfaced with a PC. SEM images were obtained using a JEOL JSM-7001F. Raman spectroscopy was performed by alpha 300 (WITEC).

GO suspension was synthesized from graphite powder by a modified Hummers method. ${ }^{23}$ Graphite powder $(3 \mathrm{~g}$ ) was taken in an Erlenmeyer flask and stirred in ice bath. $\mathrm{NaNO}_{3}$ $(1.5 \mathrm{~g})$ and concentrated $\mathrm{H}_{2} \mathrm{SO}_{4}(69 \mathrm{~mL})$ were added into the Erlenmeyer flask. Then $\mathrm{KMnO}_{4}(9 \mathrm{~g})$ was added slowly and the mixture was stirred for $3 \mathrm{~h} .120 \mathrm{~mL}$ of deionized (DI) water was added slowly over $30 \mathrm{~min}$. DI water $(200 \mathrm{~mL})$ was added, and followed by slow addition of $30 \% \mathrm{H}_{2} \mathrm{O}_{2}$ (3 $\mathrm{mL}$ ) solution. The color of the suspension changed from brown to yellow. The suspension was centrifuged at 4500 rpm with $5 \% \mathrm{HCl}$ solution for 2 rounds and then centrifuged at high speed for several times with distilled water.

Prior to the surface modification, gold coated glass electrodes were cleaned by using piranha solution $\left(\mathrm{H}_{2} \mathrm{SO}_{4}: \mathrm{H}_{2} \mathrm{O}_{2}\right.$ $=3: 1$ ), washed with sufficient amount of deionized water, and finally dried with $\mathrm{N}_{2}$ gas. In order to create the positive and negative charged layers in sequence, the freshly cleaned gold electrodes were soaked in $10 \mathrm{mM}$ MPA and $0.1 \%$ PEI solution for $12 \mathrm{~h}$ and $1.5 \mathrm{~h}$, respectively. The positively charged electrodes were immersed in $0.5 \mathrm{mg} / \mathrm{mL}$ solution of GO for $1 \mathrm{~h}$. The blocking DNA was treated on the GOmodified electrodes to prevent non-specific binding of $\mathrm{NH}_{2}-$ terminated TBA. 1:1 mixture of $200 \mathrm{mM}$ EDC and $50 \mathrm{mM}$ NHS was exposed to the GO modified surface for $1 \mathrm{~h}$ to immobilize $\mathrm{NH}_{2}$-terminated TBA. Then the surface was incubated with $1 \%$ BSA solution in PBS for $1 \mathrm{~h}$ to block nonspecific binding of thrombin on the surface.

The modified electrodes were exposed to various concentrations of thrombin solutions and incubated for $1.5 \mathrm{~h}$. For negative control experiment, same procedure was followed without thrombin. All electrochemical measurements were carried out by using electrochemical cell consisting of the modified gold electrode as the working electrode, and $\mathrm{Ag} /$ $\mathrm{AgCl}$ and $\mathrm{Pt}$ wire as the reference and counter electrodes, respectively. $\mathrm{CV}$ measurements were performed range from 
$-0.4 \mathrm{~V}$ to $-1.3 \mathrm{~V}$. EIS measurements were recorded between $10 \mathrm{kHz}$ and $0.05 \mathrm{~Hz}$ at the formal potential of $\mathrm{K}_{4}\left[\mathrm{Fe}(\mathrm{CN})_{6}\right] /$ $\mathrm{K}_{3}\left[\mathrm{Fe}(\mathrm{CN})_{6}\right]$ (1 mM, 1:1 molar ratio) in PBS. A Randle equivalent circuit was used to fit the obtained impedance spectra, which were then represented as a Nyquist plot in the complex plane.

Acknowledgments. This work was supported by Incheon National University Research Grant in 2010.

\section{References}

1. Wang, J. Biosensors and Bioelectronics 2006, 21, 1887.

2. Schedin, F.; Geim, A.; Morozov, S.; Hill, E.; Blake, P.; Katsnelson, M.; Novoselov, K. Nat. Mater. 2007, 6, 652.

3. Prabakar, S.; Hwang, Y. H.; Bae, E. G.; Shim, S.; Kim, D.; Lah, M. S.; Sohn, K. S.; Pyo, M. Adv. Mater. 2013, 25, 3307.

4. Dreyer, D. R.; Park, S.; Bielawski, C. W.; Ruoff, R. S. Chem. Soc. Rev. 2010, 39, 228.

5. Sun, X.; Liu, Z.; Welsher, K.; Robinson, J. T.; Goodwin, A.; Zaric, S.; Dai, H. Nano Res. 2008, 1, 203.

6. Zhu, Y.; Murali, S.; Cai, W.; Li, X.; Suk, J. W.; Potts, J. R.; Ruoff, R. S. Adv. Mater. 2010, 22, 3906.

7. Compton, O. C.; Nguyen, S. T. Small 2010, 6, 711.

8. Zhang, J.; Zhang, F.; Yang, H.; Huang, X.; Liu, H.; Zhang, J.; Guo, S. Langmuir 2010, 26, 6083.
9. Wang, Y.; Li, Z.; Wang, J.; Li, J.; Lin, Y. Trends in Biotechnol. 2011, 29, 205

10. Loh, K. P.; Bao, Q.; Eda, G.; Chhowalla, M. Nat. Chem. 2010, 2, 1015.

11. Haque, A.-M. J.; Park, H.; Sung, D.; Jon, S.; Choi, S.-Y.; Kim, K. Anal. Chem. 2012, 84, 1871.

12. Park, H.; Kim, J.-Y.; Hague, A. J.; Choi, S.-Y.; Kim, T.-H; Hong, J.-D.; Kim, K. Bull. Kor. Chem. Soc. 2012, 33, 4219.

13. Bonanni, A.; Chua, C. K.; Zhao, G.; Sofer, Z. K.; Pumera, M. ACS Nano 2012, 6, 8546.

14. Park, H.; Hwang, S.-J.; Kim, K. Electrochem. Commun. 2012, 24 , 100.

15. Choi, D.; Jeong, H.; Kim, K. Analyst 2014, 139, 1331.

16. Kelly, J. A.; Feigon, J.; Yeates, T. O. J. Mol. Biol. 1996, $256,417$.

17. Krauss, I. R.; Merlino, A.; Giancola, C.; Randazzo, A.; Mazzarella, L.; Sica, F. Nucleic Acids Research. 2011, 39, 7858.

18. Altintas, Z.; Uludag, Y.; Gurbuz, Y.; Tothill, I. Anal. Chim. Acta 2012, 712, 138 .

19. Gao, Y.; Kyratzis, I. Bioconjugate Chem. 2008, 19, 1945.

20. Liu, J.; Li, Y.; Li, Y.; Li, J.; Deng, Z. J. Mater. Chem. 2010, 20 , 900

21. Li, X.; Shen, L.; Zhang, D.; Qi, H.; Gao, Q.; Ma, F.; Zhang, C. Biosensors and Bioelectronics 2008, 23, 1624.

22. Cai, H.; Lee, T. M.-H.; Hsing, I. Sensors and Actuators B 2006, $114,433$.

23. Hummers, W. S., Jr.; Offeman, R. E. J. Am. Chem. Soc. 1958, 80, 1339. 